University of Nebraska - Lincoln

DigitalCommons@University of Nebraska - Lincoln

2-15-2004

\title{
Three-dimensional high-resolution reconstruction of polarization in ferroelectric capacitors by piezoresponse force microscopy
}

\section{B. J. Rodriguez}

North Carolina State University, Raleigh, brian.rodriguez@ucd.ie

Alexei Gruverman

University of Nebraska-Lincoln, agruverman2@unl.edu

A. I. Kingon

North Carolina State University, Raleigh, angus_kingon@brown.edu

R. J. Nemanich

North Carolina State University, Raleigh

J. S. Cross

Fujitsu Laboratories Ltd, Atsugi, 243-0197, Japan

Follow this and additional works at: https://digitalcommons.unl.edu/physicsgruverman

Part of the Physics Commons

Rodriguez, B. J.; Gruverman, Alexei; Kingon, A. I.; Nemanich, R. J.; and Cross, J. S., "Three-dimensional high-resolution reconstruction of polarization in ferroelectric capacitors by piezoresponse force microscopy" (2004). Alexei Gruverman Publications. 28.

https://digitalcommons.unl.edu/physicsgruverman/28

This Article is brought to you for free and open access by the Research Papers in Physics and Astronomy at DigitalCommons@University of Nebraska - Lincoln. It has been accepted for inclusion in Alexei Gruverman Publications by an authorized administrator of DigitalCommons@University of Nebraska - Lincoln. 


\title{
Three-dimensional high-resolution reconstruction of polarization in ferroelectric capacitors by piezoresponse force microscopy
}

\author{
B. J. Rodriguez, A. Gruverman, ${ }^{\text {a) }}$ A. I. Kingon, and R. J. Nemanich \\ North Carolina State University, Raleigh, North Carolina 27695 \\ J. S. Cross \\ Fujitsu Laboratories Ltd, Atsugi, 243-0197, Japan
}

(Received 22 October 2003; accepted 12 November 2003)

\begin{abstract}
A combination of vertical and lateral piezoresponse force microscopy (VPFM and LPFM, respectively) has been used to map the out-of-plane and in-plane polarization distribution, respectively, of (111)-oriented $\mathrm{Pb}(\mathrm{Zr}, \mathrm{Ti}) \mathrm{O}_{3}$-based (PZT) ferroelectric patterned and reactively-ion-etched capacitors. While VPFM and LPFM have previously been used to determine the orientation of the polarization vector in ferroelectric crystals and thin films, this is the first time the technique has been applied to determine the three-dimensional polarization distribution in thin-film capacitors and, as such, is of importance to the implementation of nonvolatile ferroelectric random access memory. Sequential VPFM and LPFM imaging have been performed in poled 1 $\times 1.5 \mu \mathrm{m}^{2}$ PZT capacitors. Subsequent quantitative analysis of the obtained piezoresponse images allowed the three-dimensional reconstruction of the domain arrangement in the PZT layers of the capacitors. It has been found that the poled capacitors, which appear as uniformly polarized in VPFM, are in fact in a polydomain state as is detected by LPFM and contain $90^{\circ}$ domain walls. Despite the polycrystallinity of the PZT layer, regions larger than the average PZT grain size are found to have the same polarization orientation. This technique has potential for clarifying the switching behavior and imprint mechanism in micro- and nanoscale ferroelectric capacitors. (C) 2004 American Institute of Physics. [DOI: 10.1063/1.1638889]
\end{abstract}

\section{INTRODUCTION}

Ferroelectric random access memory (FRAM) is considered to be faster and more durable than existing nonvolatile memory technologies and has been the subject of intense investigation. ${ }^{1}$ FRAM density has increased over the past several years, and, recently, several companies have announced production FRAM chips in the Mb range. However, in order to compete with existing memory technologies, FRAM density must continue to increase. Some of the challenges being faced in increasing FRAM density are intrinsic capacitor-to-capacitor variations resulting from the polycrystalline ferroelectric films having fewer grains per capacitor as the lateral and vertical dimensions are scaled down. In addition, reliability issues associated with FRAM bit polarization loss and imprint are always of concern.

With the development of piezoresponse force microscopy $(\mathrm{PFM})^{2-10}$ it became possible to perform direct, nondestructive, high-resolution investigations of the switching behavior of ferroelectrics in the nanoscale regime, which made PFM a useful tool for characterization of high-density FRAM capacitors. Among the accomplishments of the PFM approach is an insight into spatial variability issues of ferroelectric capacitors. ${ }^{8-12}$ In addition, the role of mechanical stress in the spatial inhomogeneity of switching behavior in ferroelectric capacitors has recently been emphasized by PFM. ${ }^{10,11}$ To better understand a nanoscale mechanism of

\footnotetext{
a) Author to whom correspondence should be addressed; electronic mail: alexei_gruverman@ncsu.edu
}

switching in polycrystalline films it would be helpful to reconstruct the three-dimensional domain arrangement at the subgrain level. Detection of only the normal component of polarization vector as is usually done in PFM is not enough to accomplish this task. Therefore, it becomes imperative to measure both the vertical and the lateral component of polarization. In this study, we undertake the three-dimensional high-resolution reconstruction of the polarization vectors in (111)-oriented $\mathrm{Pb}(\mathrm{Zr}, \mathrm{Ti}) \mathrm{O}_{3}$ ferroelectric capacitors by detecting the in-plane and out-of-plane polarization components using PFM. This approach may allow us to gain further insight into imprint and switching behavior of ferroelectric capacitors.

\section{EXPERIMENTAL DETAILS}

In this study, $200 \mathrm{~nm}$ thick layers of (111)-oriented PZT with $\mathrm{Zr} / \mathrm{Ti}$ ratio $40 / 60$ and with dopants of $\mathrm{Ca}, \mathrm{Sr}$, and $\mathrm{La}$ were sputtered onto a $\mathrm{Pt}$ bottom electrode. Transmission electron microscopy revealed a columnar grain structure with an average grain size of approximately $200 \mathrm{~nm}$. Reactive ion etching was used to fabricate $1 \times 1.5 \mu \mathrm{m}^{2}$ capacitors with 50 $\mathrm{nm}$ thick $\mathrm{IrO}_{2}$ top electrodes on the PZT surface. The PZT surface was etched $40 \mathrm{~nm}$ below the top electrode/PZT interface, leaving PZT capacitor inlands. Vertical PFM (VPFM) and lateral PFM (LPFM) imaging methods, used to detect out-of-plane and in-plane polarization components, respectively, have been described in detail elsewhere. ${ }^{5-7,11-18}$ The modulation voltage (typically $0.6 \mathrm{Vrms}$ at $10 \mathrm{kHz}$ ) was applied to the top electrode of the capacitor using a conduc- 
tive PFM tip, which was also used to detect the piezoelectric response of the capacitor. Commercially available Pt-coated Si rectangular cantilevers (1.0-2.6 N/m force constant) have been used in this study.

In PFM, an ac voltage is applied between the probing tip and a sample, which deforms due to the converse piezoelectric effect. This deformation is sensed by the cantilever. The perpendicular cantilever vibration is associated with out-ofplane polarization (VPFM), while the lateral vibration is related to in-plane polarization (LPFM). In LPFM, the $10 \mathrm{kHz}$ component of the lateral (or friction) force signal is measured by the lock-in amplifier just as the $10 \mathrm{kHz}$ component of the A-B signal is measured in VPFM. LPFM works by detecting piezoelectric shear deformations that result from any in-plane polarization component. ${ }^{13-18}$ The shear deformation, via frictional force, leads to the torsional vibration of the cantilever at the modulation frequency. For in-plane polarization, the amplitude and phase of this vibration is linked to the polarization magnitude and direction, respectively, via the $d_{15}$ piezoelectric coefficient [for a (001)-oriented grain]. Note that the tip does not have to raster in order to measure the LPFM signal (only a modulation voltage needs to be applied), unlike a friction force signal, which can only be measured if the tip is scanning.

In VPFM, the piezoresponse signal is always measured along a direction normal to the plane of the film. If the polarization vector of a ferroelectric domain is also normal to the plane of the film, the detected signal is proportional to the $d_{33}$ coefficient only, which can be accurately measured. ${ }^{5}$ However, if there is an angle between normal to the film plane and the polarization vector, the effective piezoelectric coefficient $d_{z z}$ measured in VPFM is not equal to $d_{33}$ nor is it proportional to spontaneous polarization. ${ }^{19-23}$ This is especially a concern when dealing with polycrystalline ferroelectric thin films with randomly oriented grains or with polarization fixed at an angle from the direction normal to the surface.

For tetragonal thin films, the $d_{z z}$ is given by

$$
d_{z z}(\theta)=\left(d_{31}+d_{15}\right) \sin ^{2} \theta \cos \theta+d_{33} \cos ^{3} \theta,
$$

where $\theta$ is the angle between the measurement direction and the [001] crystallographic axis. ${ }^{23}$ An important consequence of this result is that measured piezoresponse (both in LPFM and VPFM) will depend on $d_{33}, d_{31}$, and $d_{15}$ piezoelectric coefficients. Detailed quantitative analysis of a detected PFM signal in a ferroelectric with an arbitrary orientation of polarization has been given in Ref. 23. Here, we discuss the relationship between LPFM and VPFM signals and polarization in (111)-oriented tetragonal PZT film capacitors.

In Fig. 1(a), a three-dimensional plot of the piezoelectric surface for a tetragonal PZT film with a Zr/Ti ratio of 40/60 is shown. Calculations have been made using the following thin film values for the piezoelectric coefficients: $d_{31}$ $=-59 \mathrm{pm} / \mathrm{V}, d_{15}=169 \mathrm{pm} / \mathrm{V} d_{33}=162 \mathrm{pm} / \mathrm{V} .{ }^{24}$ Figure $1(\mathrm{~b})$ shows a cross-section of this plot by the (010) plane for the particular case of a (111)-oriented film. Note that the angle between the measurement direction in VPFM (which coincides with the [111] direction) and the [001] axis is fixed. For a (111)-oriented tetragonal PZT film with a $\mathrm{Zr} / \mathrm{Ti}$ ratio of
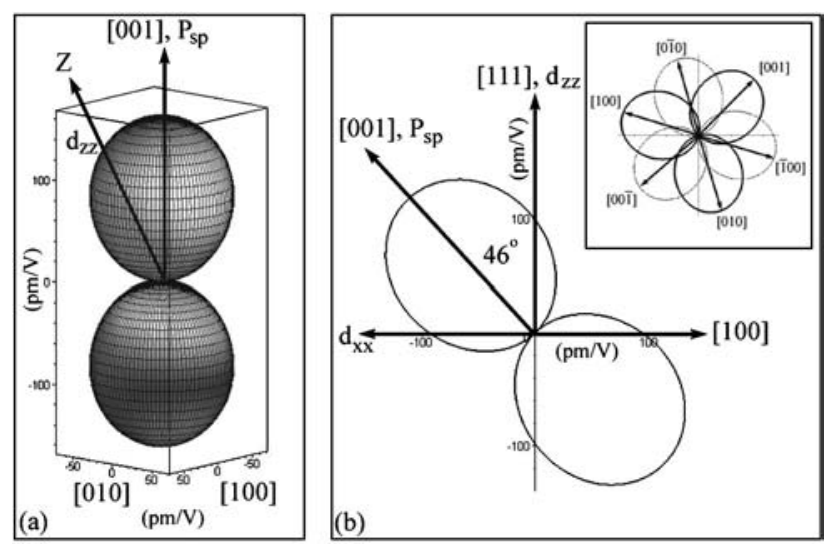

FIG. 1. (a) A three-dimensional plot of the piezoelectric surface for a tetragonal PZT film. Z indicates the measurement direction in VPFM for a randomly oriented grain. (b) Cross section of the piezoelectric surface by (010) plane for (111)-oriented tetragonal PZT film. Note that an angle between the measurement direction and the polarization direction is fixed. Inset in (b) is the projections of the piezoelectric surfaces for each of the pseudocubic principal crystallographic axes for (111)-oriented tetragonal PZT onto the $x-y$ plane, demonstrating there are six possible in-plane polarization directions.

$40 / 60$ it is equal to $\sim 46^{\circ} .{ }^{25}$ It can be also seen that with regards to VPFM, there are two possible components of polarization that can be detected.

On the other hand, in LPFM, the measurement direction is in the film plane. Therefore, the LPFM components can be found from the projection of the piezoelectric surface onto the $x-y$ plane. In the tetragonal PZT unit cell there are six possible orientations of the polarization vector (three upward and three downward along the pseudocubic principal crystallographic axes) all of which are discernible by LPFM. The inset in Fig. 1(b) shows the projections of the piezoelectric surfaces for each of the pseudocubic principal crystallographic axes onto the $x-y$ plane for (111)-oriented tetragonal PZT. In the case of a poled capacitor with domains that extend from the bottom electrode to the top electrode (through domains), the interpretation of LPFM data becomes more direct, since there are only three possible orientations of inplane polarization, each separated by $120^{\circ}$.

Generally, the scanning direction makes an arbitrary angle with the projections of the crystallographic axes onto the $x-y$ plane. To obtain complete information on the in-plane orientation of the polarization vector in a given capacitor, its LPFM images have been recorded twice. First, with the sample mounted in a known orientation, and then with the sample physically rotated by $90^{\circ}$ around film normal. In this case the effective piezoelectric coefficients $d_{z x}$ and $d_{z y}$ are measured. Note that the cantilever will only detect a shear strain for domains with an $x-y$ projection of the polarization component along the scanning direction. If the $x-y$ projection crosses both the $x$ - and $y$-axes, then there will be a component of detectible shear strain for both a measurement along the the $x$-axis and for a scan of the same area after a $90^{\circ}$ rotation of the sample. By analyzing the contrast of the LPFM amplitude images taken in two orthogonal sample orientations (proportional to $d_{z x}$ and $d_{z y}$ ) with respect to their corresponding phase contrast, it is possible to determine the 

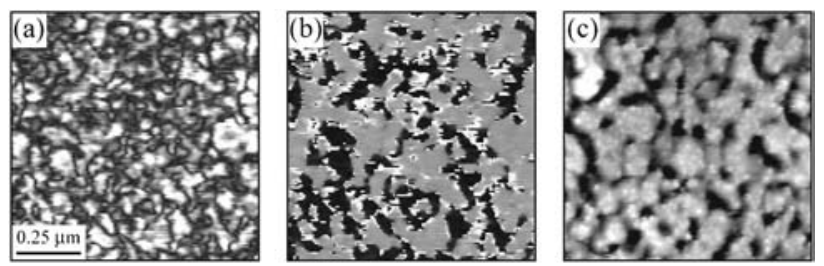

FIG. 2. LPFM (a) amplitude and (b) phase of a $1 \times 1 \mu \mathrm{m}^{2}$ region of a tetragonal (111) PZT thin film and the corresponding (c) topography.

orientation of in-plane polarization and the angle between neighboring domains. For example, if a region has bright LPFM phase contrast, maximum bright LPFM amplitude contrast, and dark LPFM amplitude contrast for an orthogonal sample orientation, the in-plane component of polarization is parallel to the initial measurement direction. Similarly, a region that has bright LPFM phase contrast and equal LPFM amplitude contrast for two orthogonal sample orientations would have the in-plane component of polarization that is exactly between the two measurement directions.

Thus, with a combination of VPFM and LPFM it is possible to perform the three-dimensional reconstruction of polarization vector.

\section{RESULTS AND DISCUSSION}

In order to better understand the domain orientation in ferroelectric capacitors, the domain structure of the underlying PZT thin film was investigated first. Figures 2(a) and 2(b) show LPFM amplitude and phase images, respectively, of the same $1 \times 1 \mu \mathrm{m}^{2}$ area. The corresponding surface topography is shown in Fig. 2(c). Regions in the LPFM phase image [Fig. 2(b)] that have dark contrast represent domains with some in-plane component of polarization oriented to the right, while bright regions represent domains with some inplane component of polarization oriented to the left. Note that while some grains have regions with different LPFM phase contrast, larger-than-grain-size regions of uniform polarization orientation are present in PZT thin films. VPFM images (not shown) revealed a random distribution of the out-of-plane component of polarization.

After PFM examination of the bare PZT surface, PFM analysis of the capacitor structures has been performed. In Figs. 3(a) and (3d), VPFM amplitude and phase are shown for a negatively poled $1 \times 1.5 \mu \mathrm{m}^{2}$ capacitor (poling has been carried out by applying a $-5 \mathrm{~V}, 3 \mathrm{~s}$ voltage pulse to the top electrode). Dark contrast in the VPFM phase image [Fig. 3(d)] represents a negative domain (with the out-of-plane component of polarization oriented upward). It is interesting to note that even though the capacitor appears to be in a single domain state as is indicated by uniform out-of-plane polarization, it does not have uniform in-plane polarization [Figs. 3(b), 3(c), 3(e), and 3(f)]. LPFM amplitude and phase images of the same capacitor taken before [Figs. 3(b) and 3(e)] and after [Figs. 3(c) and 3(f)] a counter-clockwise rotation of $90^{\circ}$ with respect to the film normal reveal a complex domain structure. In other words, even after poling, the capacitor is in fact in a polydomain state, which may impact its switching behavior. It should be noted that the topography
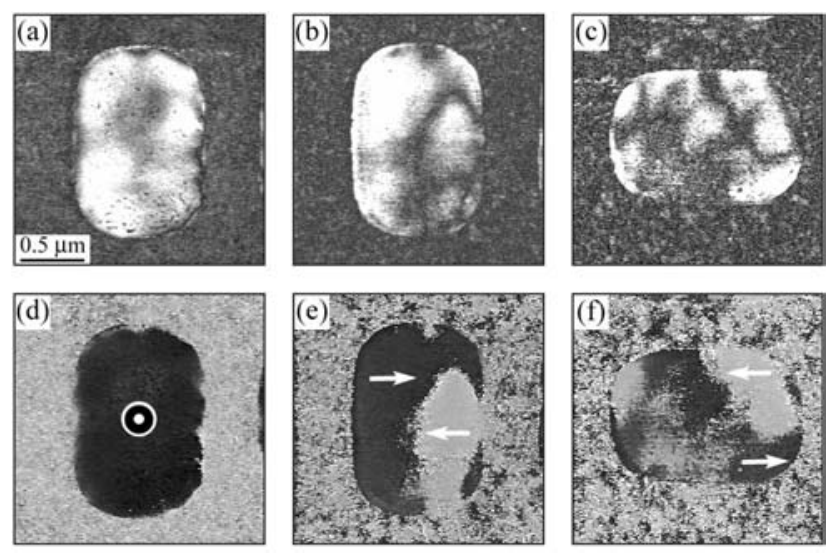

FIG. 3. VPFM (a) amplitude and (d) phase of a capacitor after application of a negative bias to the top electrode and corresponding LPFM (b) amplitude and (e) phase. LPFM (c) amplitude and (f) phase of the same capacitor following a $90^{\circ}$ rotation of the sample. Arrows in (e, f) indicate the direction of the in-plane component of polarization as deduced by LPFM for each sample orientation.

[Fig. 4(a)] has little influence on LPFM imaging so there does not appear to be an artifact related to scanning direction.

The obtained data allowed us to generate an in-plane polarization distribution map for the capacitor in Fig. 3 by overlaying the LPFM phase images for two orthogonal orientations of the sample [Fig. 4(b)]. The direction of the arrows in Fig. 4(b) were chosen based on both amplitude and phase LPFM images. If, for example, a region has dark contrast in both LPFM phase images [Figs. 3(e) and 3(f)], then the arrow (and hence the direction of the in-plane component of the spontaneous polarization) is determined to be down and to the right in the reconstructed polarization map [Fig. 4(b)]. However, if the LPFM magnitude [brighter contrast in Figs. 3(b) and 3(c)] is larger for one of the LPFM amplitude images, then the LPFM phase for that region is weighted more in determining the orientation of the region. In Fig. 4(c) an isometric cross section of the PZT capacitor structure and three-dimensional reconstruction of polarization is presented for clarity. Figure 4(d) presents the corresponding $x-y$ projection of the piezoelectric surfaces for the negative outof-plane polarization. Each arrow in Fig. 4(b) corresponds to one of the main crystallographic axes in the piezoresponse surface projection in Fig. 4(d). Note that this analysis has been performed by assuming a single-crystalline PZT film, which is of course not the case. In a polycrystalline film, one might expect there to be an arbitrary angle between the inplane components of polarization of adjacent grains. Anyhow, the reconstructed map of the in-plane polarization of the capacitor in Fig. 4(b) reveals domains with the lateral size larger than the average grain size. This is consistent with the LPFM data obtained on the bare PZT surface. This is an interesting result: even though the film is polycrystalline, it behaves as if it were a single-crystalline. Since pole-figure analysis did not reveal any in-plane crystallographic ordering we have to assume a presence of the low angle grain texture at the submicron scale.

In Fig. 4(b) there is a region denoted by " $x$ " which does not have one of the three possible orientations of polariza- 

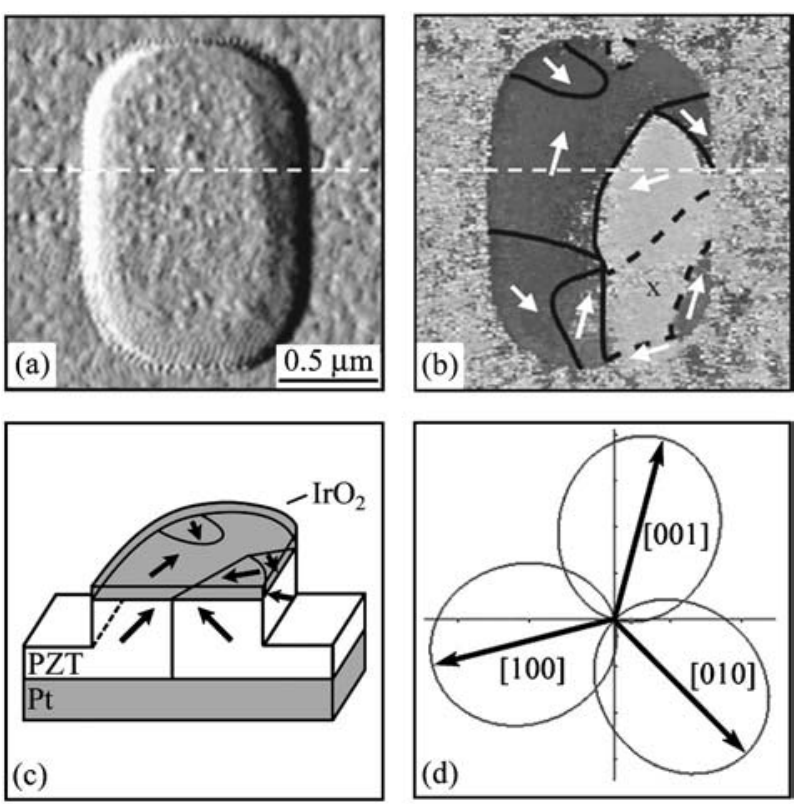

FIG. 4. (a) Topography and (b) reconstruction of the in-plane polarization map of the negatively poled (111) PZT ferroelectric capacitor shown in Fig. 3. (c) Isometric cross section of the PZT capacitor structure with threedimensional reconstruction of polarization. (d) Projection of the piezoelectric surfaces onto the (111) plane for three possible in-plane polarization directions for the negatively poled capacitor. $90^{\circ}$ domain walls are indicated with a solid line in (b), while indeterminate boundaries are marked with a dashed line. Note that due to the presence of the top electrode, it is impossible to determine if these domain walls coincide with grain boundaries or run through the grain interior.

tion. However, this region is also marked by reduced piezoresponse amplitude, suggesting the presence of non-through domains and is furthermore located near several domain boundaries. Therefore, the polarization direction cannot be determined reliably.

Domain walls are classified as two types: those with antiparallel polarizations on either side of the wall $\left(180^{\circ}\right.$ domain walls), and those separating domains with polarization vectors that differ by $90^{\circ}\left(90^{\circ}\right.$ domain walls). In PFM, $90^{\circ}$ domain walls can be seen as separating adjacent domains with opposite LPFM and uniform VPFM phase contrasts. These types of domain walls are illustrated by solid lines in Fig. 4(b). Unfortunately, due to the presence of the top electrode, it is impossible to find out if the domain walls coincide with the grain boundaries or run through the grain interior. In Fig. 5, VPFM and LPFM amplitude and phase images are presented for another ferroelectric capacitor with nonuniform out-of-plane polarization. Ideally, it should be possible to determine if $180^{\circ}$ domain walls are present near the region denoted "y" in Fig. 5(d). The presence of non-through domains, however, as revealed by reduced VPFM amplitude in Fig. 5(a), makes distinguishing between $90^{\circ}$ and $180^{\circ}$ domain walls challenging.

In our previous study, ${ }^{11}$ it has been found that the inner regions of several of the capacitors are negatively imprinted and tend to switch back after being poled by a positive bias, while regions at the edge of the capacitors tend to exhibit more symmetric hysteresis behavior. However, as can be seen in VPFM amplitude images of the negatively poled ca-
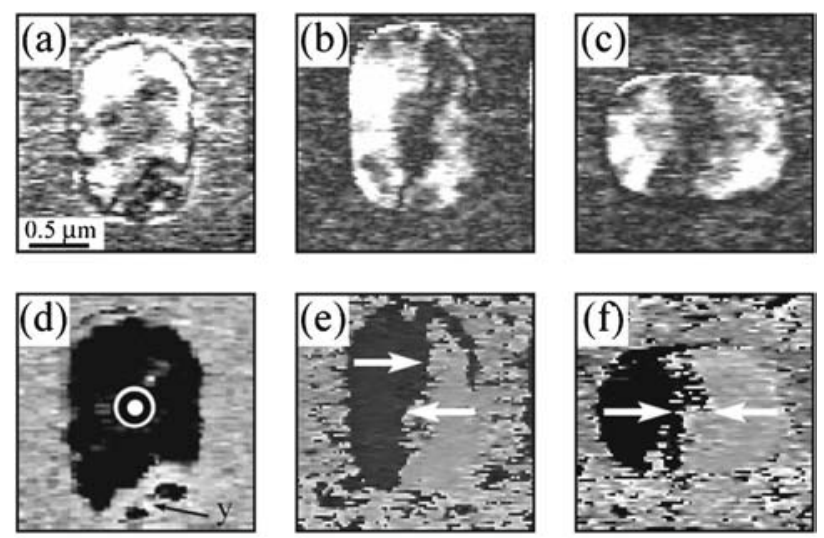

FIG. 5. VPFM and LPFM images of (a, b) amplitude and (d, e) phase, respectively, of a ferroelectric capacitor with nonuniform out-of-plane polarization. LPFM (c) amplitude and (f) phase of the same capacitor following a $90^{\circ}$ clockwise rotation of the sample. The presence of adjacent positive and negative domains in the region marked " $y$ " and also along the edge of the capacitor suggests the existence of $180^{\circ}$ domain walls. However, the possibility of non-through domains makes an exact determination of the domain wall type difficult.

pacitors [Figs. 3(a) and 5(a)] there appears to be domain boundaries running along the edges of the capacitors at a distance of less than $100 \mathrm{~nm}$ from the edge. These boundaries separate the edge regions with positive out-of-plane polarization from the capacitor interior with negative polarization. Although from local piezoresponse loop measurements these along-the-edge regions were found to be switchable, ${ }^{11}$ they cannot be completely erased by application of an external negative bias, as spontaneous backswitching likely occurs after the voltage is removed. Trapped charges could lead to the existence of charged domain walls in the interior of the capacitors, which could contribute to their imprint behavior. Further work is underway to identify these charged domain walls and their role in imprint using three-dimensional reconstruction of polarization by a combination of VPFM and LPFM.

\section{SUMMARY}

A combination of vertical and lateral PFM revealed the domain orientation of the underlying PZT thin film and allowed mapping of the three-dimensional polarization of (111)-oriented $1 \times 1.5-\mu \mathrm{m}^{2}$ PZT capacitors. It has been found that the poled capacitors, which appear as uniformly polarized in VPFM, are in fact in a polydomain state as is detected by LPFM. Despite the polycrystallinity of the PZT layer, regions larger than the average PZT grain size are found to have the same polarization orientation, suggesting a presence of the low angle grain texture at the submicron scale. This technique has potential for clarifying the capacitor size effect and electrode edge effect on imprint and switching in ferroelectric capacitors.

\section{ACKNOWLEDGMENTS}

The authors acknowledge financial support of the National Science Foundation (Grant No. DMR-0235632) and 
Fujitsu Limited throughout this project. The authors thank Professor J.-P. Maria for performing pole figure analysis of the PZT film used in this study.

${ }^{1}$ O. Auciello, J. F. Scott, and R. Ramesh, Phys. Today 1998, 22.

${ }^{2}$ A. Gruverman, O. Auciello, and H. Tokumoto, Appl. Phys. Lett. 69, 3191 (1996).

${ }^{3}$ T. Hidaka, T. Maruyama, I. Sakai, M. Saitoh, L. A. Wills, R. Hiskes, S. A. Dicarolis, and J. Amano, Integr. Ferroelectr. 17, 319-327 (1997).

${ }^{4}$ A. Gruverman, O. Auciello, and H. Tokumoto, Annu. Rev. Mater. Sci. 28, 101 (1998).

${ }^{5}$ J. A. Christman, R. R. Woolcott, A. I. Kingon, and R. J. Nemanich, Appl. Phys. Lett. 73, 3851 (1998).

${ }^{6}$ J. A. Christman, S.-H. Kim, H. Maiwa, J.-P. Maria, B. J. Rodriguez, A. I. Kingon, and R. J. Nemanich, J. Appl. Phys. 87, 8031 (2000).

${ }^{7}$ S. V. Kalinin and D. A. Bonnell, Phys. Rev. B 65, 125408 (2002).

${ }^{8}$ S. Hong, E. L. Colla, E. Kim, D. V. Taylor, A. K. Tagantsev, P. Muralt, K. No, and N. Setter, J. Appl. Phys. 86, 607 (1999).

${ }^{9}$ M. Alexe, C. Harnagea, D. Hesse, and U. Gösele, Astrophys. Appl. Phys. Lett. 79, 242 (2001)

${ }^{10}$ I. Stolichnov, E. Colla, A. Tagantsev, S. Bharadwaja, S. Hong, N. Setter, J. S. Cross, and M. Tsukada, Appl. Phys. Lett. 80, 4804 (2002).

${ }^{11}$ A. Gruverman, B. J. Rodriguez, A. I. Kingon, R. J. Nemanich, J. S. Cross, and M. Tsukada, Appl. Phys. Lett. 82, 3071 (2003).

${ }^{12}$ A. Gruverman, B. J. Rodriguez, A. I. Kingon, R. J. Nemanich, J. S. Cross, and M. Tsukada, Appl. Phys. Lett. 83, 728 (2003).
${ }^{13}$ L. M. Eng, H.-J. Güntherodt, G. Rosenman, A. Skliar, M. Oron, M. Katz, and D. Eger, J. Appl. Phys. 83, 5973 (1998).

${ }^{14}$ L. M. Eng, M. Abplanalp, P. Günter, Appl. Phys. A: Mater. Sci. Process. A66, S679-S683 (1998).

${ }^{15}$ M. Abplanalp, L. M. Eng, and P. Günter, Appl. Phys. A: Mater. Sci. Process. A66, S231-S234 (1998).

${ }^{16}$ L. M. Eng, H. J. Güntherodt, G. A. Schneider, U. Köpke, and J. Muñoz Saldaña, Appl. Phys. Lett. 74, 233 (1999).

${ }^{17}$ A. Roelofs, U. Böttger, R. Waser, F. Schlaphof, S. Trogisch, and L. M. Eng, Appl. Phys. Lett. 77, 3444 (2000).

${ }^{18}$ C. S. Ganpule, V. Nagarajan, B. K. Hill, A. L. Roytburd, E. D. Williams, S. P. Alpay, A. Roelofs, R. Waser, and L. M. Eng, J. Appl. Phys. 91, 1477 (2002).

${ }^{19}$ X. Du, U. Belegundu, and K. Uchino, Jpn. J. Appl. Phys., Part 1 36, 5580 (1997).

${ }^{20}$ X. Du, J. Zheng, U. Belegundu, and K. Uchino, Appl. Phys. Lett. 72, 2421 (1998).

${ }^{21}$ X. Du, Q.-M. Wang, U. Belegundu, A. Bhalla, and K. Uchino, Mater. Lett. 40, 109 (1999).

${ }^{22}$ S. Kalpat, X. Du, I. R. Abothu, A. Akiba, H. Goto, and K. Uchino, Jpn. J. Appl. Phys., Part 1 40, 713 (2001).

${ }^{23}$ C. Harnagea, A. Pignolet, M. Alexe, and D. Hesse, Integr. Ferroelectr. 38, 23 (2001).

${ }^{24}$ M. J. Haun, Ferroelectrics 99, 63 (1989).

${ }^{25}$ G. Shirane, K. Suzuki, and A. Takeda, J. Phys. Soc. Jpn. 7, 12 (1952). 